\title{
Bioanalysis
}

\section{Outsourcing in bioanalysis: a CRO perspective}

\begin{abstract}
Steve Lowes from $\mathrm{Q}^{2}$ Solutions speaks to Sankeetha Nadarajah, Managing Commissioning Editor: about outsourcing strategy implementation. Steve started his industrial career at VG Biotech in the UK that became the LC-MS instrument entity of Waters Corporation. Since joining the CRO group that became Advion and then $\mathrm{Q}^{2}$ Solutions, his career has focused on regulated bioanalysis with particular emphasis on LC-MS. He is a founding member of the Global Bioanalysis Consortium and a pastchair of the AAPS Bioanalytical Focus Group. At $Q^{2}$ Solutions, Steve leads the scientific disciplines around LC-MS bioanalysis for both small molecule and biomolecule applications including biomarker assays. Steve has over 40 peer-reviewed publications on bioanalysis and is a frequent speaker at national and international conferences.
\end{abstract}

\section{Q Could you tell Bioanalysis a little about your career to date?}

My introduction to bioanalysis started with GC/MS in the late 1980s. I was fortunate enough to catch the attention of Professor Malcolm Rose at what is now Sheffield Hallamshire University in the UK (then Sheffield City Polytechnic). Professor Rose had already established a solid reputation for himself in MS with the text, Mass Spectrometry for Chemists and Biochemists [1]. However, what really drew me into $\mathrm{MS}$ was a $\mathrm{PhD}$ program sponsored by the Royal Society of Chemistry and supervised by Professor Rose. The program focus was to investigate the development of a GC/MS derivatization method for urinary acylcarnitines to help study inborn errors of metabolism with particular emphasis on sudden infant death syndrome. I am not sure we even referred to my PhD studies as bioanalysis at the time, but detecting, differentiating and quantifying compounds of interest in a complex biological matrix, using MS had me hooked. Transitioning from the formalities of completing an undergraduate degree to using a powerful analytical technique to solve real-world problems and have positive impact on human health was a formative experience that initiated my career in bioanalysis. I followed Professor Rose to The Open University (Milton Keynes, UK), where I completed my PhD studies, and then I took my first job at Fisons Instruments/ VG BioTech (Manchester, UK). That timing (early 1990s) coincided with the rapid evolution of interfacing liquid chromatography to quadrupole MS. By working in the applications laboratory at the time, I got a rapid education in the strengths and weaknesses of thermospray, dynamic fast-atom bombardment, particle beam and eventually enjoyed early success with the atmospheric pressure ionization techniques of atmospheric pressure chemical ionization (APCI), electrospray and IonSpray ${ }^{\mathrm{TM}}$. After experiencing the tremendous technology developments of LC-MS in the 1990s at VG BioTech (subsequently became Micromass and then Waters), I emigrated from the UK to join Advanced Bioanalytical Services, a 2-year-old startup CRO company (NY, USA). What became Advion and subsequently Quintiles/Q ${ }^{2}$ Solutions exposed me to regulated bioanalysis and the impact it has on modern drug development. Over 25 years and the field continues to evolve, advance and present new challenges that have influenced my career to date. I am very thankful for the opportunities that

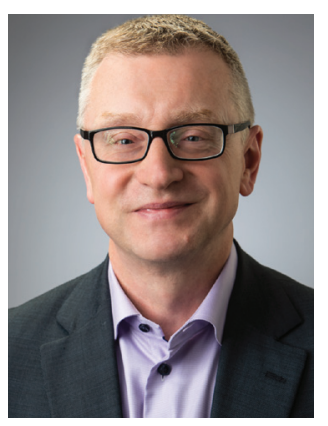

Stephen Lowes Bioanalytical \& ADME Laboratories, Q2 Solutions, 19 Brown Road, Ithaca, NY 14850, USA

stephen.lowes@q2labsolutions.com 
have come with this progress and the exciting future prospects bioanalysis still presents.

With your years of experience in the field, how would you say bioanalytical outsourcing has evolved over the last 20 years?

Bioanalytical outsourcing is certainly not the same as 20 years ago. In the early to mid-1990s, the focus in bioanalysis was on small-molecule drugs and the impact LC-MS technology was having on the field. There was an ongoing transition from LC-UV and GC/MS that required an investment in new technology and expertise, but otherwise the barrier to entry was relatively low. As such, those with LC-MS experience and one or two instruments could garner attention and be capable of serving the market. Relationships with Pharma bioanalysis experts were critical to any new laboratory though, because the confidence was not there that LC-MS could be easily or reliably done by a CRO. In fact, most of the bioanalysis work was not outsourced at all, but rather conducted by heavily resourced internal laboratories at the major pharmaceutical companies. As such, the outsourcing market was much smaller and differentiated on the basis of technical capabilities and reputation. However, just as the Pharma industry has gone through fundamental changes over the last 20 years, the bioanalysis CRO industry has adapted accordingly. Today, most regulated bioanalysis is outsourced, and it has also broadened in scope. Technical capabilities and needs are no longer focused only around routine small-molecule LC-MS. Of course biologic drugs have become an increased proportion of drug-development pipelines, but additional drug modalities, biomarkers, sample-collection practices, sample matrices, required sensitivity and evolving global regulatory requirements have all had influence on bioanalytical outsourcing.

The advances in bioanalysis technology over the last 20 years has been huge, and in turn this has impacted the outsourcing industry. Advances in sensitivity and ease of use of the triple quadrupole instruments have tracked alongside the increased outsourcing of smallmolecule bioanalysis. Similarly, we now see technology improvements impacting the ligand-binding assay (LBA) work with increased emphasis on automation or automation-assisted workflows. The technology improvements have enabled increased capacity while attenuating the proportional costs of such growth through efficiencies and necessary skill sets to get the work done. I do think we are now moving into a phase of technology development that opens new potential that in some ways is reminiscent of the early days of atmospheric pressure ionization LC-MS. Some emphasis is again turning to technical hands-on capability around sample preparation and low-flow chromatography coupled to newer MS detection platforms and data handling processes.

Parallel to the technology and capability improvements, we have witnessed increasing regulatory needs. Much of this makes sense and the development of regulatory language has promoted increased confidence and demonstrable assurances of data accuracy. This has been most effective when open consultation with the bioanalytical community has taken place as part of the process. Nonetheless, the influence of global health authorities on what and how regulated bioanalysis is conducted today is a significant contrast to 20 years ago. It will be interesting how this continues as the science and technology change at a seemingly everincreasing pace.

Q Would you say there has been much change in how outsourcing is done in the last 3-5 years?

I think there has been a shift to the preferred provider model by many tasked with outsourcing bioanalytical work. That is, sponsors select a short list (typically $<5)$ of bioanalytical providers that have the quality, capability, capacity and pricing to meet the projected needs. Breadth of capabilities and capacity favor the larger bioanalytical CROs in such circumstances. This tends to be the model for the larger (and now somewhat consolidated) global pharmaceutical companies. Opportunities still exist for smaller bioanalytical laboratories, but they tend toward either niche areas of expertise or other unique aspects that can meet the sponsor's specific needs.

\section{Q How would you describe the current state of} play in bioanalysis outsourcing?

Bioanalysis outsourcing is continuing to increase in terms of volume and scope. There is a sponsor expectation that a CRO will conduct bioanalysis to the quality and regulatory standards as would be done by an in-house laboratory. In return, the CROs are investing in the relationships with the sponsors to support future business. These synergistic relationships are supported by something of a migration of bioanalysis key opinion leaders from Pharma companies to the CROs. The technical and experience strengths of the outsource providers now often compare with those of the sponsors, and we see this play out in various ways. One notable example is the dialog regulatory authorities have with bioanalytical CROs through industry consortia and focus groups. I see these as healthy developments that make for good opportunities and careers in both Pharma/biotech and CRO organizations. 
Q Do you have any key recommendations for the sponsors \& the CROs, considering today's status? The interdependencies of the sponsor and the CROs require capabilities on both sides of the relationship and excellent communication between parties. As the industry evolves to address new therapeutic areas and modes of treating disease, collaborative approaches to how the bioanalysis phase will be conducted will be important. With a wealth of bioanalytical expertise resident in CROs, it will be prudent to leverage it effectively. My recommendation here is to ensure the bioanalytical talent is appropriately preserved by both the sponsor and the CRO. While leveraging capacity toward the CRO side of the relationship makes strategic sense, a lack of knowledge or appreciation of the bioanalytical science on the sponsor's side may expose a weakness in the process. Bioanalysts best address challenges when they are working as a team sharing information and experience from early discovery and development through all of the clinical phases.

\section{Q How do you believe that a CRO is able to} effectively manage costs but still maintain productivity \& quality of work?

Without going into confidential business strategy, the obvious key resources for any bioanalytical laboratory are the people and the technology employed. Both require continuous investment. It would be convenient if regulated bioanalysis was taught at the university level with a practical and significant laboratory experience component. Prior to postgraduate studies, however, this is rarely the case and so it is incumbent on the CRO to efficiently train the staff. Most of us will point to on-the-job training that has primarily influenced our bioanalysis expertise and this is not likely to change. However, increasing options and material are now available to help teach bioanalysis. Taking advantage of some newer texts and online training courses is an excellent means of helping improve capabilities of a bioanalytical laboratory. Supplementing this should be strong internal training programs that share and leverage internal and external expertise.

As to technology developments, we are all accustomed to managing data and sample information in bioanalytical laboratory information management system. What we are seeing now is the implementation and routine use of electronic laboratory notebooks (ELNs). These will supplement the laboratory information management system, and I do believe will improve documented compliance and help reduce human errors. I think it is still to be proven how much cost and productivity efficiencies are gained, and to be fair, this will be dependent on what you are comparing against and the specific design attributes of a given
ELN product. Nonetheless, cost benefits are typically an associated expectation of an ELN initiative.

Beyond the ELN, I see lots of other technology developments happening that drive to improve capability, productivity and quality. Automation assistance in sample preparation through robotic liquid handlers is not new but continues to develop in capability. Of course, standardizing automation is a challenge in bioanalysis since each bioanalytical method is unique to a given compound. Most have found the 'automation solution' needs to be taken judiciously, but 96-well plate approaches have helped productivity, and I anticipate further technology solutions in sample preparation will feature strongly in the future of bioanalysis.

\section{Q Where do you see bioanalytical outsourcing} heading in the next few years \& what challenges do you expect to emerge?

In some respects, my answer to this question relates to the last question in terms of bioanalytical outsourcing needing to drive increased productivity, demonstrate quality and adapt to new requirements. The increase in biologic drug pipelines will influence the CRO capabilities with LBA skill sets continuing to be in high demand. I do not see this being restricted to traditional LBA approaches. Hybrid immunoaffinity/LC-MS is now established as a valid approach to biotherapeutic and protein biomarker quantitation. This is still transitioning to CROs, but I believe it will continue to grow as a complement to LBA bioanalysis.

Another MS technology development that I see CROs adopting is high-resolution mass spectrometry (HRMS) coupled to LC. The selectivity gains possible with HRMS are profoundly impressive, and I see these instrument platforms figuring prominently in CROs with increasing demand from the sponsors.

Synonymous with, but not exclusive to HRMS instruments and hybrid immunoaffinity/LC-MS, I see microflow and nanoflow LC separations becoming a standard offering in bioanalytical outsourcing. This comes from the drive to increase assay sensitivity which these techniques can deliver. We have had a continuous thirst for additional sensitivity as part of our bioanalytical discipline. We continue to strive for assay sensitivity, but not at the expense of selectivity - hence the combination of optimum analyte isolation, efficient chromatographic separations and selective detection. In some respects, we need to think of all these standard attributes of a bioanalytical assay in a holistic manner. The decision of how to extract the analyte of interest will likely influence the chromatography choice, which in turn, will dictate the detection platform. This can be a challenge for the bioanalytical laboratory that has evolved around small molecule analysis. Taking the 
approach of a generic sample preparation (e.g., protein precipitation) combined with conventional chromatography (e.g., $2.1 \mathrm{~mm}$ diameter, $\mathrm{C} 18$ reversed-phase LC column) and selective reaction monitoring, triple quadrupole MS detection will not be sufficient for the new challenges of biologic bioanalysis.

Another development that I predict will continue to present to the bioanalytical laboratory is microsampling. European bioanalysts have adapted to microsampling in a manner that demonstrates practical utility. The progress being demonstrated is likely to continue to influence the bioanalysis discipline as a whole with dry and liquid microsampling in various configurations becoming accepted strategy across a wide variety of preclinical and clinical needs. Outsourcing laboratories will need to adapt accordingly.

My concluding expectation is around endogenous analytes and biomarkers. While much of the bioanalysis we conduct today can be accommodated in established regulatory language, biomarkers present some fundamental challenges that we are only starting to debate. To conduct guidance-driven bioanalysis on protein biomarkers in particular needs significantly more thought and dialog with the regulatory agencies. I believe this will happen, but it will push

\section{Reference}

1 Johnstone RAW, Rose ME. Mass Spectrometry for Chemists and Biochemists Cambridge University Press, Cambridge; NY, USA (1996). us out of the comfort zone of prescriptive approaches and fully initiate fit-for-purpose bioanalysis. Coming back to the mentioned need for expertise at the sponsor and the outsourcing sides of the relationships, the communication will likely also extend to the regulators. Establishing customized bioanalysis strategy with collaborative acceptance and support ahead of experimental work and data generation/ submission may become a common approach in the future.

\section{Disclaimer}

The opinions expressed in this interview are those of the interviewee and do not necessarily reflect the views of Future Science Ltd or Q2 Solutions.

\section{Financial \& competing interests disclosure}

The author of this article is employed by a CRO with financial interests in outsourcing bioanalysis. The author has no other relevant affiliations or financial involvement with any organization or entity with a financial interest in or financial conflict with the subject matter or materials discussed in the manuscript apart from those disclosed.

No writing assistance was utilized in the production of this manuscript. 